\title{
Lethal Effects of Aspergillus niger against Mosquitoes Vector of Filaria, Malaria, and Dengue: A Liquid Mycoadulticide
}

\author{
Gavendra Singh and Soam Prakash \\ Environmental and Advanced Parasitology and Vector Control Biotechnology Laboratories, Department of Zoology, Faculty of Science, \\ Dayalbagh Educational Institute, Dayalbagh, Agra 282005, India \\ Correspondence should be addressed to Soam Prakash, prakashsoamdei@gmail.com
}

Received 20 October 2011; Accepted 9 January 2012

Academic Editor: Tatiana Scorza

Copyright ( 92012 G. Singh and S. Prakash. This is an open access article distributed under the Creative Commons Attribution License, which permits unrestricted use, distribution, and reproduction in any medium, provided the original work is properly cited.

\begin{abstract}
Aspergillus niger is a fungus of the genus Aspergillus. It has caused a disease called black mold on certain fruits and vegetables. The culture filtrates released from the A. niger ATCC 66566 were grown in Czapek dox broth (CDB) then filtered with flash chromatograph and were used for the bioassay after a growth of thirty days. The result demonstrated these mortalities with $\mathrm{LC}_{50}$, $\mathrm{LC}_{90}$, and $\mathrm{LC}_{99}$ values of Culex quinquefasciatus 0.76, 3.06, and 4.75, Anopheles stephensi 1.43, 3.2, and 3.86, and Aedes aegypti $1.43,2.2$, and $4.1 \mu \mathrm{l} / \mathrm{cm}^{2}$, after exposure of seven hours. We have calculated significant $\mathrm{LT}_{90}$ values of Cx. quinquefasciatus 4.5 , An. stephensi 3.54, and Ae. aegypti 6.0 hrs, respectively. This liquid spray of fungal culture isolate of $A$. niger can reduce malaria, dengue, and filarial transmission. These results significantly support broadening the current vector control paradigm beyond chemical adulticides.
\end{abstract}

\section{Introduction}

Rapidly emerging insecticide resistance is creating an urgent need for new active ingredients to control the adult mosquitoes [1]. A range of isolates belonging to the fungal species Metarhizium anisopliae and Beauveria bassiana have been shown to infect and significantly reduce the longevity of adult Anopheles mosquitoes, killing them within 14 days [2-4]. The Beauveria bassiana has infected mosquitoes of the insecticide resistant Anopheles arabiensis at two different temperatures [5]. The fungi have been applied by spraying on mosquitoes with an oil formulation of infectious spores. The fungal spores begin pathogenic and invade the mosquitoes, after which the fungus multiplies and kills its host within two weeks [2]. Similarly, the isolates of Metarhizium anisopliae ICIPE-30 and Beauveria bassiana I93-825 (IMI 391510) have reduced mosquito survival on immediate exposure and up to 28 days after application [6]. The critics have argued that "slow acting" these fungal biopesticides is, therefore, incapable of delivering mosquito control in different parts of the world. The entomopathogenic fungi can be integrated into control programmes additional information regard- ing isolate selection, optimisation of production, and formulation is required. While many successful laboratory evaluations of the efficacy of entomopathogenic fungi have been conducted [2, 7-9]. Therefore, more research on fungal formulations and evaluating of various formulations, delivery techniques remains essential against mosquitoes.

Aspergillus niger is a filamentous ascomycete fungus that is ubiquitous in the environment and has been implicated in opportunistic infections of humans [10]. A. niger is most widely known for its role as a citric acid producer [11]. With production of citric acid at over one million metric tons annually, A. niger citric acid production serves as a model fungal fermentation process. This organism is a soil saprobe with a wide array of hydrolytic and oxidative enzymes involved in the breakdown of plant lignocellulose. A variety of these enzymes from A. niger is important in the biotechnology industry. The A. niger is also an important model organism for several important research areas including the study of eukaryotic protein secretion in general, the effects of various environmental factors on suppressing or triggering the export of various biomass degrading enzymes, molecular mechanisms critical to fermentation process development, 


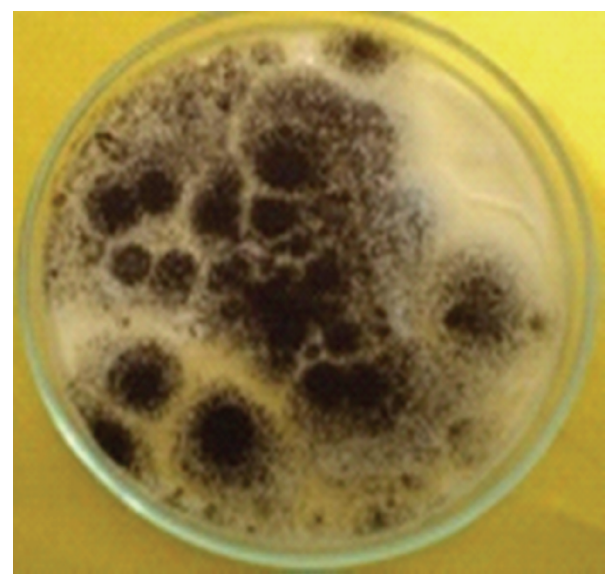

(a)

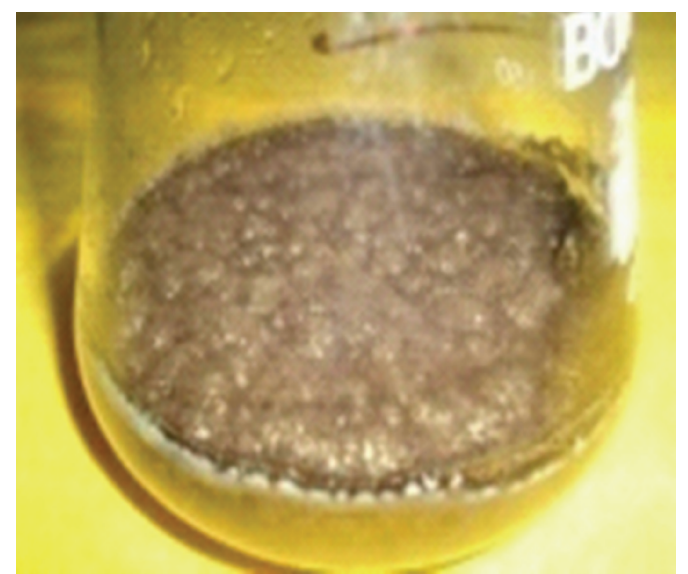

(b)

Figure 1: The cultures of Aspergillus niger: (a) solid medium on Czapek dox agar (CDA), (b) liquid medium Czapek dox broth maintained in the laboratory.

and mechanisms involved in the control of fungal morphology. These are encouraging characteristics which encourage for further research on mosquitoes control.

Mosquito vectors are solely responsible for transmitting diseases, such as malaria, dengue, chikungunya, Japanese encephalitis, yellow fever, and lymphatic filariasis [12]. Malaria is an important cause of death and illness in children and adults, especially in tropical countries. Malaria is caused by a parasite that is transmitted from one human to another by the bite of infected Anopheles stephensi. Half of the world's population is at risk from malaria. Each year almost 250 million cases occur, causing 860000 deaths [13]. Approximately 3.5 billion people live in dengue endemic countries which are located in the tropical and subtropical regions of the world [14]. Lymphatic filariasis, commonly known as elephantiasis, is so far a neglected tropical disease. The infection occurs when filarial parasites are transmitted to humans through Culex quinquefasciatus. More than 1.3 billion people in eighty-one countries worldwide are threatened by lymphatic filariasis [15]. In the present investigation, we have reported the lethal effects of purified culture filtrates of $A$. niger against An. stephensi, Cx. quinquefasciatus, and Ae. aegypti in the laboratory.

\section{Materials and Methods}

2.1. Collection and Culture of Aspergillus niger. The strain of Aspergillus niger (ATCC 66565) was obtained from Microbial Type Culture Collection and Gene Bank (MTCC 2587) Institute of Microbial Technology, Chandigarh, India. A. niger was maintained on autoclaved Czapek dox broth (sucrose: $30.0 \mathrm{~g}$, sodium nitrate: $3.0 \mathrm{~g}$, dipotassium phosphate: $1.0 \mathrm{~g}$, magnesium sulphate: $0.05 \mathrm{~g}$, potassium chloride: $0.05 \mathrm{~g}$, ferrous sulphate: $0.01 \mathrm{~g}$, deionized water: $1000 \mathrm{~mL}$ ) and adjusts $\mathrm{pH}$ 6.5. The broth was supplemented with $50 \mu \mathrm{g} / \mathrm{mL}$ chloramphenicol as a bacteriostatic agent. The colonies of $A$. niger were grown on Czapek dox agar (CDA), solid medium plates were transferred to each flask using an inoculation needle.
The conical flasks, inoculated with $A$. niger, were incubated at $25^{\circ} \mathrm{C}$ for 30 days (Figure 1 ).

\subsection{Preparation of Flash Chromatograph Columns and Fil-} tration. In the Flash chromatograph, a plastic column was filled with silica gel, with the sample to be separated placed on top of this support. The rest of the column is filled with an isocratic or gradient solvent which, with the help of pressure, enables the sample to run through the column and become separated. Flash chromatography used air pressure initially to speed up the separation. The culture filtrates were obtained by filtering the broth through Whatman no.1 filter paper. These metabolites were further filtered with the flash chromatograph.

2.3. Bioassays. The flash chromatograph purified culture filtrates were used for bioassays against laboratory reared $C x$. quinquefasciatus, Ae. aegypti, and An. stephensi as per the standard procedures recommended by World Health Organization with some modifications [16]. The freshly emerged three-day-old sugar fed adults were used for the assay. The five different volumes of $1.6,2.2,2.7,3.2$, and $3.8 \mu \mathrm{L} / \mathrm{cm}^{2}$ of metabolites were sprayed in a cage $(25 \mathrm{~cm}$ length $\times$ $15 \mathrm{~cm}$ width $\times 5 \mathrm{~cm}$ depth) containing 25 mosquitoes. The exposed mosquitoes were kept under observation, and dead mosquitoes were discarded daily. Each bioassay including control was conducted in triplicate on different days. In the control cages deionized water was sprayed. Daily mortality counts were performs. The bioassays were carried out at room temperature with $75 \pm 5 \%$ relative humidity. The negative control was deionized water with $1 \% \mathrm{CDB}$ while the positive control was Gokilaht-S 5EC (d,d-trans-cyphenothrin).

2.4. Statistical Analysis. The efficacy study of the filtrate metabolites of $A$. niger was assessed against $C x$. quinquefasciatus, Ae. aegypti, and An. stephensi by probit analysis [17] with the statistical package IBM SPSS 19.0. 


\section{Results and Discussion}

In the present observations, we have evaluated the lethal effects of culture filtrates of $A$. niger against adult mosquitoes. The lethal effects of $A$. niger with $\mathrm{LC}_{50}, \mathrm{LC}_{90}$, and $\mathrm{LC}_{99}$ values of $C x$. quinquefasciatus were $0.76,3.06$, and $4.751 \mu \mathrm{L} / \mathrm{cm}^{2}$. Moreover, in case of the An. stephensi it was observed as $1.43,3.2$, and 3.86. While in case of Ae. aegypti it was recorded as $1.43,2.2$, and $4.1 \mu \mathrm{L} / \mathrm{cm}^{2}$. These values were calculated after the exposure of seven hours along with their probit quotations (Table 1). The entomopathogenic fungus has been successfully reduceing mosquito vectors population in laboratories and field trials $[2-4,18]$. The fungal infections for the mosquitoes become increasingly sick and are eventually killed, but the process can take up to a week or more. The adult mosquitoes pick up the fungal spores when resting on treated surfaces.

Unlike fast-acting chemical neurotoxins, fungal pathogens do not cause rapid mortality or immediate "knockdown" but rather act over a number of days as the fungal spores penetrate the insect cuticle and then proliferate within the hemocoel [19]. The A. clavatus has been found highly pathogenic against larvae of Ae. aegypti, Cx. quinquefasciatus, and An. gambiae [20]. The mortality rates were $100 \%$ against both $A$ e. aegypti, and Cx. quinquefasciatus, while against An. gambiae it was $95 \%$ after 24 hours. The entomopathogenic fungus $B$. bassiana has used as an alternative vector control tool against insecticide-resistant mosquitoes under conditions typical of indoor resting environments [5]. A range of fungal-based insecticide combinations was used to test effects of timing and sequence of exposure. Both the laboratory-reared and field-collected mosquitoes were highly resistant to permethrin but susceptible to $B$. bassiana and $M$. anisopliae infection, inducing $100 \%$ mortality within nine days. Simultaneous coexposure induced the highest mortality, up to $70.362 \%$ for a combined Beauveria and permethrin exposure within a time range of one gonotrophic cycle (4 days) [21]. In present investigation the lethal time effect of $A$. niger with $\mathrm{LT}_{50}$ and $\mathrm{LT}_{90}$ values of $C x$. quinquefasciatus 2.57, 4.5, An. stephensi 1.58, 3.54, and Ae. aegypti 1.65, $6.0 \mathrm{hrs}$ were calculated (Table 1). At the first time for increase in percent mortalities, a combination of an insecticide and an entomopathogenic fungus has been tested against Ae. aegypti. It can be an alternative to applications of high concentrations of chemical insecticides. The Ae. aegypti could be controlled by surface application of entomopathogenic fungi and that the efficiency of these fungi increased by combining the fungi with ultra-low concentrations of insecticides, resulting in higher mortality following relatively short exposure times [22].

This study distinctly demonstrates that the A. niger culture filtrates have induced a higher impact on adult mosquitoes with significant percent of mortalities (Figure 2). The applied concentrations have affected $C x$. quinquefasciatus, An. stephensi, and Ae. aegypti with relevant $\mathrm{LC}_{50}, \mathrm{LC}_{90}$ and $\mathrm{LC}_{99}$ values after exposure of seven hours (Figure 3 ). The recorded lethal effects show the potential for integrated fungus control measures to dramatically reduce malaria, filarial, and dengue vectors. The pathogenic fungi produce a wide

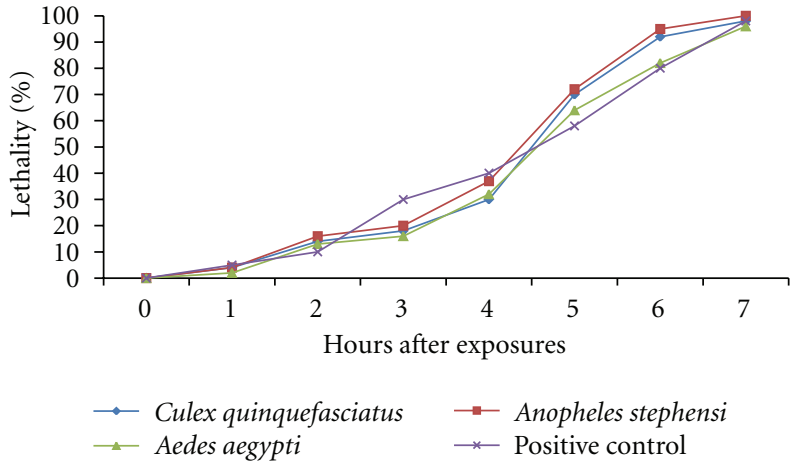

FIGURE 2: Effect of culture filtrates of Aspergillus niger against Culex quinquefasciatus, Anopheles stephensi, and Aedes aegypti after exposure of 7 hours.

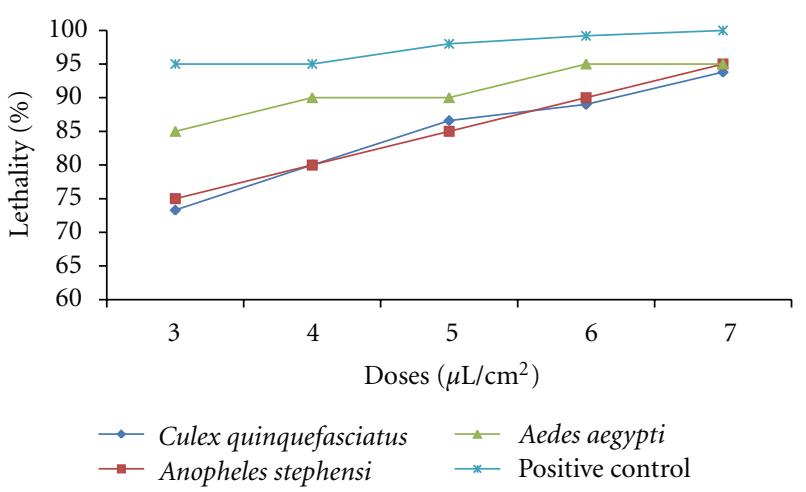

Figure 3: Effect of culture filtrates of Aspergillus niger against Culex quinquefasciatus, Anopheles stephensi and Aedes aegypti at different concentrations.

variety of toxic metabolites, which vary from low molecular weight products of secondary metabolism to complex cyclic peptides and proteolytic enzymes [23]. A significant progress has been made in understanding enzymes involved with the penetration of host cuticle and the role of mosquitocidal toxins. The fungal metabolites can be more effective by joint action of numerous toxins and enzymes.

The A. niger is the best producer of extracellular lipase [24]. The present study shows that the A. niger purified fungal culture filtrates have enhanced their lethal effects against An. stephensi, Cx. quinquefasciatus, and Ae. aegypti. Moreover, the presence of mycotoxin "ochratoxin" in A. niger can be fast-acting metabolites for control of adult mosquitoes. Ideally, all these new findings could be implemented with a time application with its fast acting impact against $A n$. stephensi, Cx. quinquefasciatus, and Ae. aegypti populations. This investigation can be further improved by implementing enhanced fungus-based strategy to control the adult population. In our laboratories, Trichophyton ajelloi, Chrysosporium lobatum, C. tropicum, Lagenidium giganteum, Culicinomyces clavisporus, and Fusarium oxysporum have so far been successfully screened and were found pathogenic against larvae and adults of An. stephensi, Cx. quinquefasciatus, and Ae. aegypti. These fungal strains have shown lethal effect after exposure of 


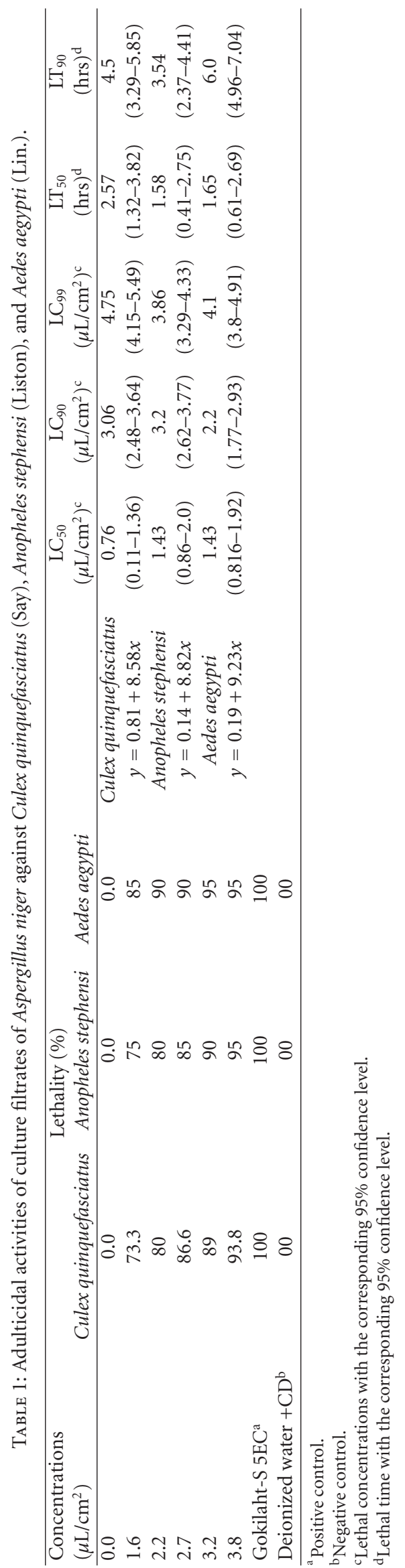


24, 42, and 72 hours. Consequently, A. niger has found effective in very short time. This unique property of this fungus requires further field testing in different climatic zones.

\section{Conflict of Interests}

The authors do not have any conflict of interests.

\section{Acknowledgments}

The authors thank M/s. Sumitomo Chemicals India Pvt. Ltd. for supply of Gokilaht-S 5EC. They thanking Prof. V. G. Das, Director, Dayalbagh Educational Institute, Agra for his encouragements. They are also thankful to the University Grants Commission, New Delhi, of Major Research Project (MRP/Soam Prakash) for the financial support 2010-2012 and to DST-FIST program (2003-2008) for providing laboratory facilities. G. Singh is indebted to UGC, New Delhi, for an award of Postdoctoral Fellowship (2009-2011).

\section{References}

[1] S. Blanford, W. Shi, R. Christian et al., "Lethal and pre-lethal effects of a fungal biopesticide contribute to substantial and rapid control of malaria vectors," PLoS ONE, vol. 6, no. 8, article e23591, 2011.

[2] S. Blanford, B. H. K. Chan, N. Jenkins et al., "Fungal pathogen reduces potential for malaria transmission," Science, vol. 308, no. 5728, pp. 1638-1641, 2005.

[3] E. J. Scholte, B. N. Njiru, R. C. Smallegange, W. Takken, and B. G. J. Knols, "Infection of malaria (Anopheles gambiae s.s.) and filariasis (Culex quinquefasciatus) vectors with the entomopathogenic fungus Metarhizium anisopliae," Malaria Journal, vol. 2, no. 1, article 29, 2003.

[4] E. J. Scholte, K. Ng'Habi, J. Kihonda et al., "An entomopathogenic fungus for control of adult African malaria mosquitoes," Science, vol. 308, no. 5728, pp. 1641-1642, 2005.

[5] C. K. Kikankie, B. D. Brooke, B. G. J. Knols et al., "The infectivity of the entomopathogenic fungus Beauveria bassiana to insecticide-resistant and susceptible Anopheles arabiensis mosquitoes at two different temperatures," Malaria Journal, vol. 9, no. 1 , article 71, 2010.

[6] L. L. Mnyone, M. J. Kirby, D. W. Lwetoijera et al., "Tools for delivering entomopathogenic fungi to malaria mosquitoes: effects of delivery surfaces on fungal efficacy and persistence," Malaria Journal, vol. 9, no. 1, article 246, 2010.

[7] S. Prakash, G. Singh, N. Soni, and S. Sharma, "Pathogenicity of Fusarium oxysporum against the larvae of Culex quinquefasciatus (Say) and Anopheles stephensi (Liston) in laboratory," Parasitology Research, vol. 107, no. 3, pp. 651-655, 2010.

[8] G. Singh and S. Prakash, "Efficacy of Lagenidium giganteum (Couch) metabolites for control Anopheles stephensi (Liston) a malaria vector," Malaria Journal, vol. 9, supplement 2, article P46, 2010.

[9] G. Singh and S. Prakash, "Evaluation of culture filtrates of Culicinomyces clavisporus: mycoadulticide for Culex quinquefasciatus, Aedes aegypti and Anopheles stephensi," Parasitology Research, vol. 110, no. 1, pp. 267-272, 2012.

[10] J. R. Perfect, G. M. Cox, J. Y. Lee et al., "The impact of culture isolation of Aspergillus species: a hospital-based survey of aspergillosis," Clinical Infectious Diseases, vol. 33, no. 11, pp. 1824-1833, 2001.

[11] J. Magnuson and L. Lasure, "Organic acid production by filamentous fungi," in Advances in Fungal Biotechnology for Industry, Agriculture, and Medicine, J. Tkacz and L. Lange, Eds., pp. 307-340, Plenum; Kluwer Academic, New York, NY, USA, 2004.

[12] J. A. Rozendaal, Vector Control: Methods for Use by Individuals and Communities, WHO, Geneva, Switzerland, 1997.

[13] World Health Organization, "World Malaria Report," 2010.

[14] World Health Organization, "Lymphatic filariasis," 2011, http://www.who.int/mediacentre/factsheets/fs102/en/.

[15] World Health Organization, "Guidelines on the quality, safety and efficacy of dengue tetravalent vaccine (live attenuated)," WHO/DRAFT, (DEN) 1-93, May 2011.

[16] World Health Organization, "Guidelines for testing mosquito adulticides for indoor residual spraying and treatment of mosquito nets," WHO/CDS/NTD/WHOPES/GCDPP/2006.3, 2006.

[17] D. J. Finney, Probit Analysis, Cambridge University Press, Cambridge, UK, 3rd edition, 1971.

[18] S. S. Mohanty and S. Prakash, "Effects of culture media on larvicidal property of secondary metabolites of mosquito pathogenic fungus Chrysosporium lobatum (Moniliales: Moniliaceae)," Acta Tropica, vol. 109, no. 1, pp. 50-54, 2009.

[19] M. B. Thomas and A. F. Read, "Can fungal biopesticides control malaria?” Nature Reviews Microbiology, vol. 5, no. 5, pp. 377-383, 2007.

[20] F. Seye, O. Faye, M. Ndiaye, E. Njie, and J. M. Afoutou, "Pathogenicity of the fungus, Aspergillus clavatus, isolated from the locust, Oedaleus senegalensis, against larvae of the mosquitoes Aedes aegypti, Anopheles gambiae and Culex quinquefasciatus," Journal of Insect Science, vol. 9, no. 53, 2009.

[21] M. Farenhorst, B. G. J. Knols, M. B. Thomas et al., "Synergy in efficacy of fungal entomopathogens and permethrin against west african insecticide-resistant anopheles gambiae mosquitoes," PLoS ONE, vol. 5, no. 8, article e12081, 2010.

[22] A. R. Paula, A. T. Carolino, C. O. Paula, and R. I. Samuels, "The combination of the entomopathogenic fungus Metarhizium anisopliae with the insecticide Imidacloprid increases virulence against the dengue vector Aedes aegypti (Diptera: Culicidae)," Parasites and Vectors, vol. 4, no. 1, article 8, 2011.

[23] A. L. Demain and A. Fang, "The natural functions of secondary metabolites," in Advances in Biochemical Engineering Biotechnology: History of Modern Biotechnology, Th. Scheper, Ed., vol. 69, pp. 1-39, Springer, Heidelberg, Germany, 2000.

[24] G. Falony, J. C. Armas, J. C. D. Mendoza, and J. L. M. Hernández, "Production of extracellular lipase from Aspergillus niger by solid-state fermentation," Food Technology and Biotechnology, vol. 44, no. 2, pp. 235-240, 2006. 

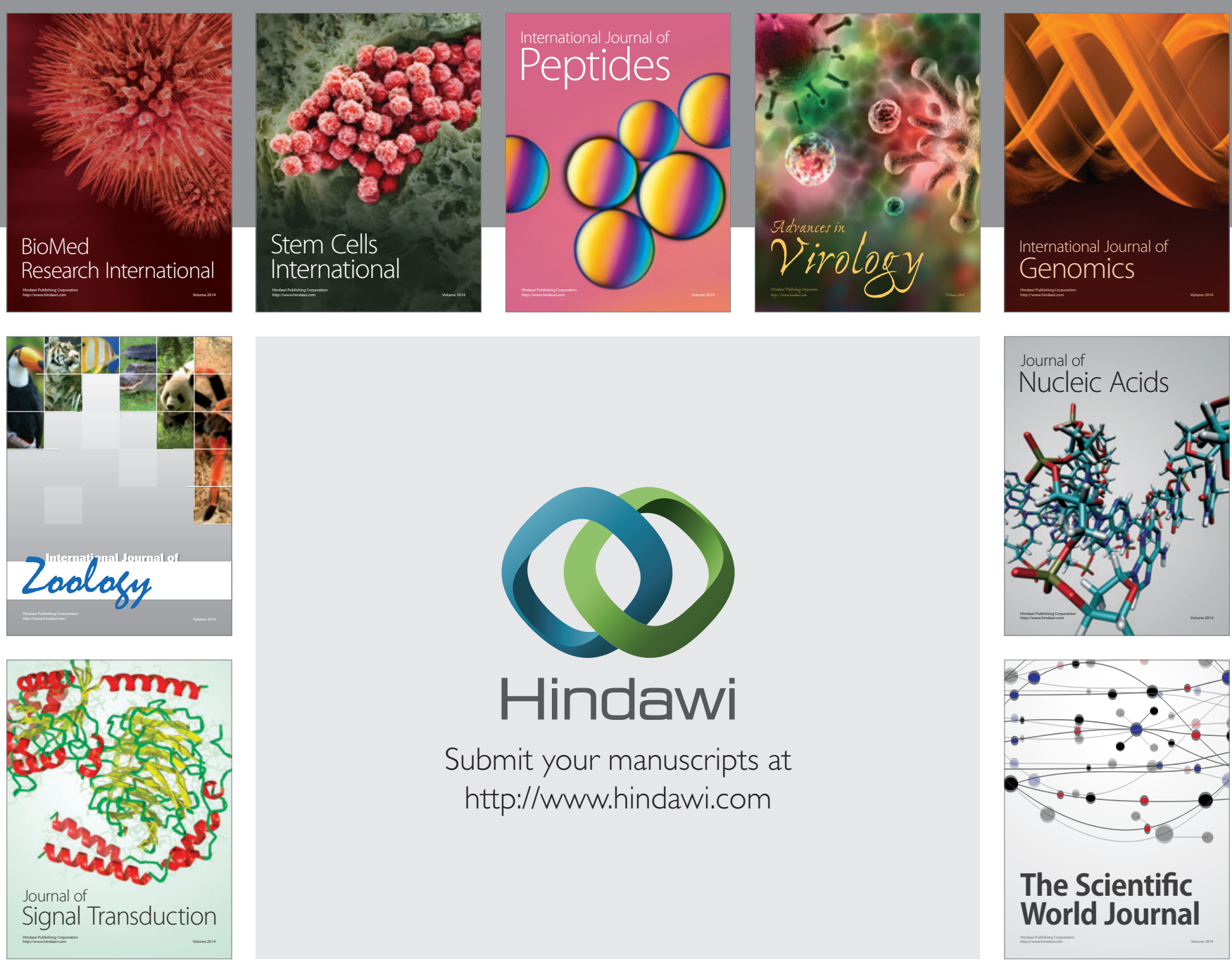

Submit your manuscripts at

http://www.hindawi.com
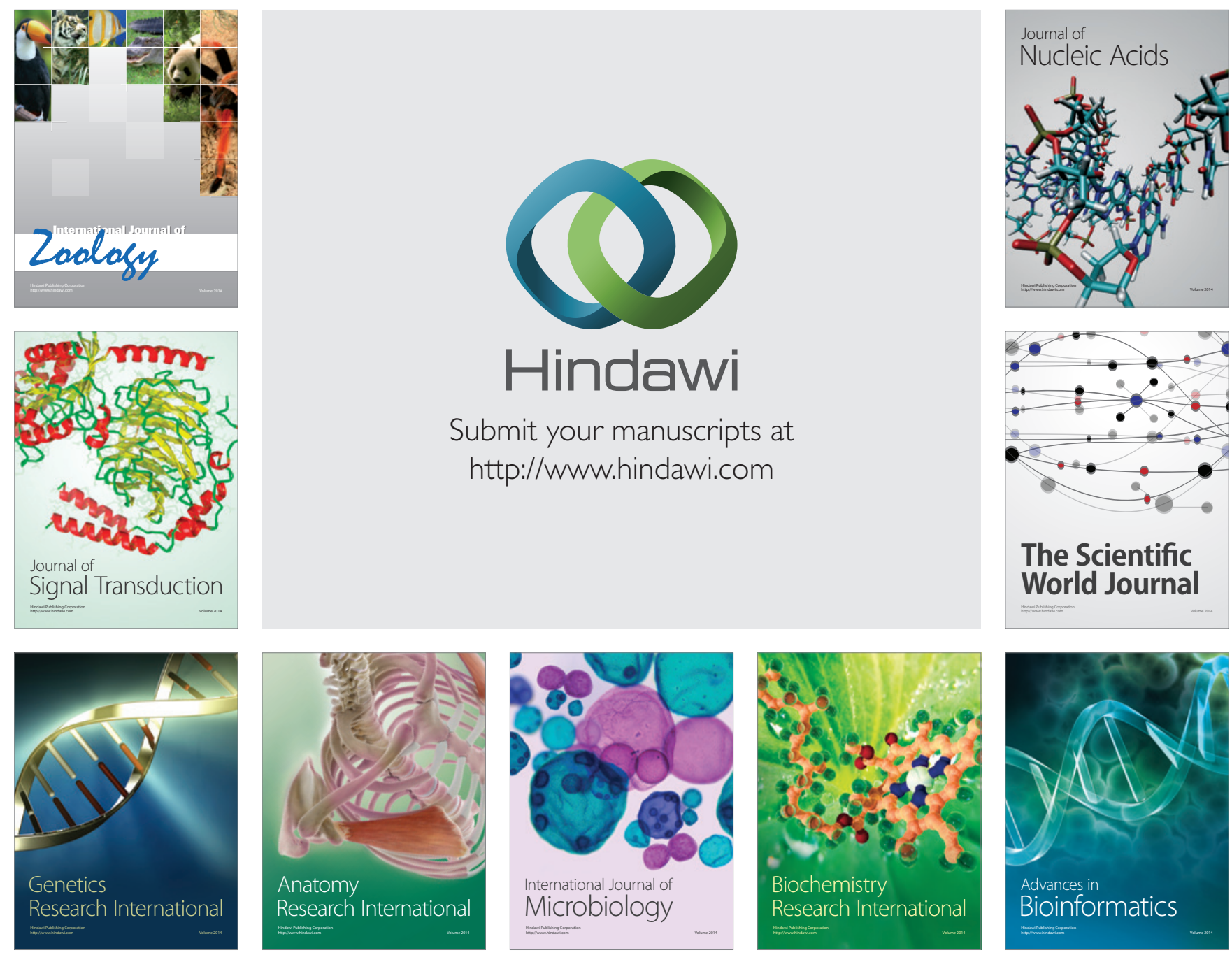

The Scientific World Journal
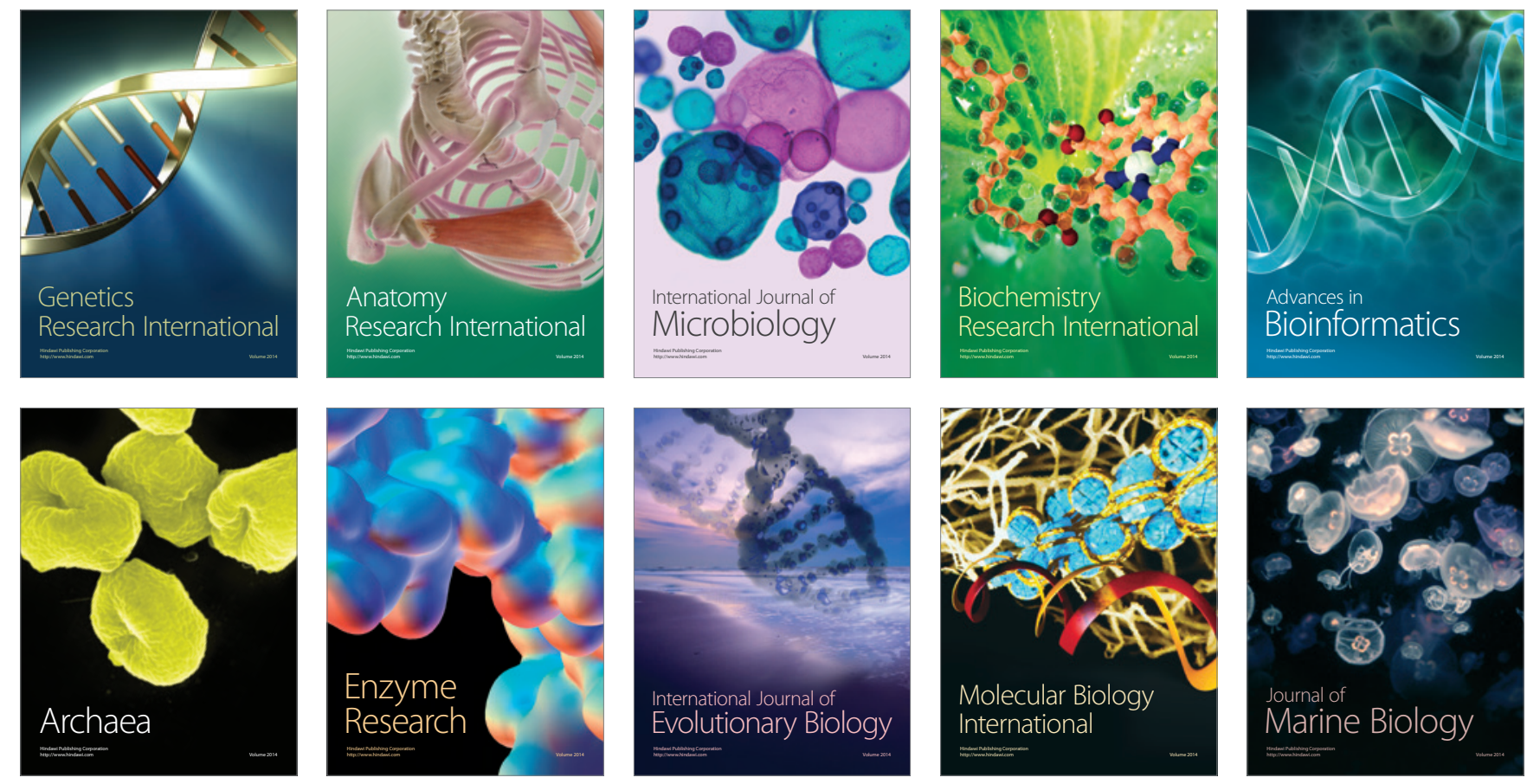